\title{
The Magnetic Control Tunable Characteristics of Localized Modes of One-dimensional Cantor Quasicrystal Structure Containing Nanoparticle Magnetic Fluid
}

\author{
C. X. ZENG ${ }^{1, a}$, J. P. LI' ${ }^{2, b}$, K. LIAO', c and Y. M. XIE ${ }^{2,3, d, *}$ \\ ${ }^{1}$ School of Science and Technology, Gannan Normal University, Ganzhou 341000, Jiangxi, China \\ ${ }^{2}$ School of Physics and Electronic Information, Gannan Normal University, Ganzhou 341000, China \\ ${ }^{3}$ Institute of Optoelectronic Materials and Technology, Gannan Normal University, Ganzhou 341000, \\ China \\ azcx6502@126.com, b gnsyljp@163.com, ${ }^{\mathrm{c}} 930488337 @ q q . c o m,{ }^{d}$ xieyingmao@126.com \\ * Corresponding author
}

Keywords: Nanoparticle Magnetic Fluid, Quasicrystal Structure, Transfer Matrix Method, Localized Modes

\begin{abstract}
The magnetic control tunable characteristics of localized modes of One-dimensional Cantor quasicrystal structure containing nanoparticle magnetic fluid is studied by using the transfer matrix method. First, the physical model of One-dimensional Cantor quasicrystal structure containing nanoparticle magnetic fluid is built. Then, the transmission spectrum of One-dimensional Cantor quasicrystals structure containing nanoparticle magnetic fluid is calculated by transfer matrix method. Finally, the magnetic control tunable characteristics of localized modes are discussed. The results show that the generation of One-dimensional Cantor quasicrystal structure containing nanoparticle magnetic fluid increased, the number of localized modes increased as well; the wavelength of localized modes shift to the long-wave direction with the refractive index of magnetic fluid increasing; the higher generation, the higher of the quality factor of the split localized modes; the wavelength drift variation decreases with the increasing of localized modes level at the same generation.
\end{abstract}

\section{Introduction}

In 1984, Shechtman and others published the report about the study of quasicrystal for the first time ${ }^{[1]}$. It has attracted the attention of researchers widely and become the research hotspot of materials science ${ }^{[2]}$. Quasicrystal structure belongs to aperiodic structure between periodic structure and disordered structure from the structural features. Compared to periodic structure, aperiodic structure has no translation symmetry; Compared to disordered structure, aperiodic structure is the ordered and certainty system. Quasiperiodic structure is a particular aperiodic structure such as Fibonacci, Cantor and Thue-Morse ${ }^{[2]}$, etc.

Scientists are interested in Cantor quasiperiodic structure, because it is a typical quasiperiodic structure. The Cantor sequence can be given by the recursive rule: $S_{n}=S_{n-1} B_{n} S_{n-1}, B_{n}=3^{n-1} B_{1}\left(n \geq 1, B_{1}=B\right)$. Starting with $\mathrm{S}_{0}=\mathrm{A}$, the Cantor generations are $\mathrm{S}_{0}=\mathrm{A}, \mathrm{S}_{1}=\mathrm{ABA}, \mathrm{S}_{2}=\mathrm{ABAB}^{3} \mathrm{ABA}$ and $\mathrm{S}_{3}=\mathrm{ABAB}^{3} \mathrm{ABAB}^{9} \mathrm{ABAB}^{3} \mathrm{ABA}^{[2]}$. Photonic crystal containing nanoparticle magnetic fluid is a kind of tunable photonic crystal ${ }^{[3,4]}$. The photonic crystal containing nanoparticle magnetic fluid has attractive application prospect on the new magnetron tunable photonic devices, causing the attention of scholars at home and abroad, and it has become a hot spot in the field of the new tunable photonic crystals because of the non-contact magnetic control adjustable features. The magnetic field control characteristics of photonic crystal fiber are studied by some scholars. The magnetic field sensor is made. For example, the research team of Nanyang technological university in Singapore fills the nanoparticle magnetic fluid of higher refractive index $(>1.45)$ in the hollow of magnetic photonic crystal fiber, based on the band gap effect of the photonic crystal fiber with magnetic fluid which studies the magnetic fiber optic sensor ${ }^{[5]}$. The colloid ferrofluid as primitive materials of one-dimensional photonic crystals by the team of Ji-ping Huang at Fudan University, which the magnetic control of one-dimensional photonic crystals is studied based on colloid ferrofluid ${ }^{[6,7]}$. The temperature tunable characteristics of photonic crystal fiber which filled with $\mathrm{Fe}_{3} \mathrm{O}_{4}$ nano-particle is studied by the 
researcher of Nankai University ${ }^{[8]}$. The hollow photonic crystal fiber Fabry - Perot sensor based on magnetic fluid of measuring magnetic field and photonic crystal fiber Bragg grating sensor filled with the magnetic fluid are studied by the researcher of Northeastern University ${ }^{[9-11]}$. In 2014, Design of multichannel filters based on the use of periodic Cantor dielectric One-dimensionals is studied by Tzu-Chyang King and others ${ }^{[12]}$. Zero measure Cantor spectra for continuum one-dimensional quasicrystal is studied by Daniel Lenz at the same year ${ }^{[13]}$.

However, there are few reports about the study of One-dimensional Cantor quasicrystal structure containing nanoparticle magnetic fluid. The magnetic control tunable characteristics of localized modes of One-dimensional Cantor quasicrystal structure containing nanoparticle magnetic fluid is studied in this paper. First, the physical model of One-dimensional Cantor quasicrystal structure containing nanoparticle magnetic fluid is built. Then, the transmission spectrum of One-dimensional Cantor quasicrystal structure containing nanoparticle magnetic fluid is calculated by transfer matrix method. Finally, the magnetic control tunable characteristics of localized modes is discussed, it has certain guiding significance for the photonic devices such as optical switch, filter in theory.

\section{Physical Model}

The third generation, fourth generation and fifth generation structures of One-dimensional Cantor quasicrystal structure containing nanoparticle magnetic fluid are $\mathrm{S}_{2}=\mathrm{ABAB}^{3} \mathrm{ABA}$, $\mathrm{S}_{3}=\mathrm{ABAB}^{3} \mathrm{ABAB}^{9} \mathrm{ABAB}^{3} \mathrm{ABA}$ and $\mathrm{S}_{4}=\mathrm{ABAB}^{3} \mathrm{ABAB}^{9} \mathrm{ABAB}^{3} \mathrm{ABAB}^{27} \mathrm{ABAB}^{3} \mathrm{ABAB}^{9} \mathrm{ABAB}^{3} \mathrm{ABA}$. $\mathrm{A}$ is Si dielectric layer that refractive index is $n_{a}=3.42$, and $\mathrm{B}$ is dielectric layer of magnetic fluid.

\section{The Results of Numerical Calculation}

The Primitive materials of One-dimensional Cantor quasicrystal structure containing nanoparticle magnetic fluid are $\mathrm{Si}$ and water-based $\mathrm{Fe}_{3} \mathrm{O}_{4}$ magnetic fluids, the optical thickness is equal to it $n_{a} * d_{a}=\bar{n}_{b} * d_{b}=\lambda_{0} / 4, \lambda_{0}$ is the center wavelength. The refractive index $n_{b}$ of magnetic liquid is related to the distribution of magnetic particles of magnetic liquid closely. There are many factors that have influence on the distribution of the magnetic particles in magnetic fluids such as the magnetic dipole interaction between particles, the Brownian motion of particles which is closely related to the temperature, extra magnetic field, capture light, etc. When the room temperature is $20^{\circ} \mathrm{C}$, wavelength of light source is $1550 \mathrm{~nm}$, density of water-based $\mathrm{Fe}_{3} \mathrm{O}_{4}$ magnetic fluids is $1.2 \mathrm{~g} / \mathrm{mL}$, the variation range of the extra magnetic field intensity is from 0 0e to $16610 \mathrm{e}$, the refractive index $n_{b}$ of magnetic liquid decreases from $n_{b h}=1.447$ to $n_{b l}=1.425$ with the increasing of magnetic field intensity. Here, water-based $\mathrm{Fe}_{3} \mathrm{O}_{4}$ magnetic fluid is selected as the primitive materials of photonic crystals.

The refractive index of dielectric layers $\mathrm{A}$ and $\mathrm{B}$ are $n_{a}, n_{b}$ and its thickness are $d_{a},{ }^{d}{ }_{b}$ respectively. Making the light wave to incident on the surface of One-dimensional Cantor quasicrystal structure containing nanoparticle magnetic fluid. The formulae which the light reflection coefficient and transmission coefficient are calculated by the transfer matrix method are[14]

$$
\begin{aligned}
& t=\frac{2 n_{0}}{n_{0}\left(m_{11}+n_{N+1} m_{12}\right)+\left(m_{21}+n_{N+1} m_{22}\right)} \\
& r=\frac{n_{0}\left(m_{11}+n_{N+1} m_{12}\right)-\left(m_{21}+n_{N+1} m_{22}\right)}{n_{0}\left(m_{11}+n_{N+1} m_{12}\right)+\left(m_{21}+n_{N+1} m_{22}\right)}
\end{aligned}
$$

where $m_{i j}$ is the matrix elements of $\mathrm{M}$

$$
M_{b}=\left(\begin{array}{cc}
\cos \delta_{b} & -\frac{i}{\eta_{b}} \sin \delta_{b} \\
-i \eta_{b} \sin \delta_{b} & \cos \delta_{b}
\end{array}\right)
$$


The reflectance $\mathrm{R}$ and the transmittance $\mathrm{T}$ respectively are

$$
\begin{aligned}
& R=|r|^{2} \\
& T=|t|^{2}
\end{aligned}
$$

Taking the parameters $n_{a}=3.42, n_{b l}=1.425, n_{b h}=1.447, \overline{\mathrm{n}}_{b}=1.4385, n_{a} * d_{a}=\bar{n}_{b} * d_{b}=\lambda_{0} / 4$, the $\lambda_{0}=1550 \mathrm{~nm}$ is the center wavelength. The third, fourth and fifth generation transmission spectrum of One-dimensional Cantor quasicrystal structure containing nanoparticle magnetic fluid are calculated by transfer matrix method when the refractive index of magnetic liquid changes with the intensity of magnetic field, results are shown in figure 1(a), figure1(b) and figure1(c).

The results of figure 1 show that: there are localized modes on the transmission spectrum of the third, fourth and fifth generation One-dimensional Cantor quasicrystal structure containing nanoparticle magnetic fluid in the band gap (from $1130 \mathrm{~nm}$ to $2400 \mathrm{~nm}$ ), and the number of localized modes are 2, 4, and 8 respectively, the number of localized modes within a certain band gap increased with the generation of One-dimensional Cantor quasicrystal structure containing nanoparticle magnetic fluid increasing. From the transmission spectrum diagram of the third and fourth generation can be found that one localized mode is split out from both sides of each localized modes of the third generation respectively, and the localized mode also can be split out from the localized modes within the band gap respectively from the fourth generation to the fifth generation.

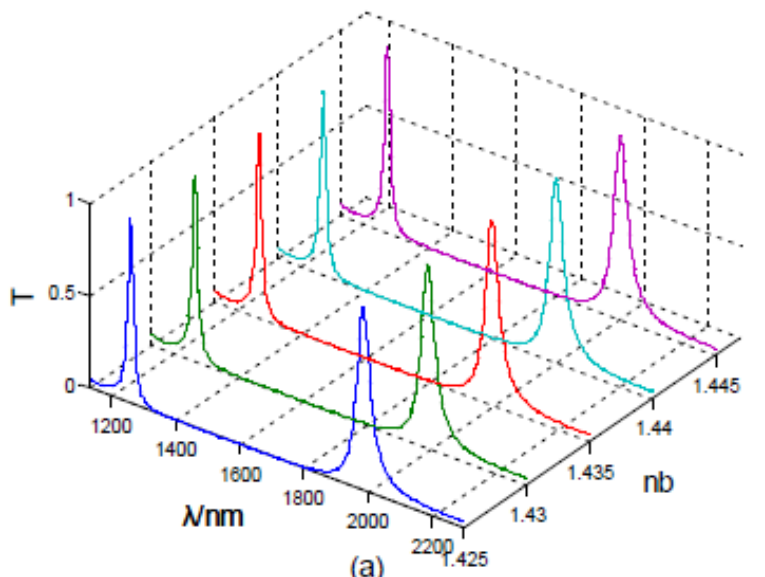

(a)

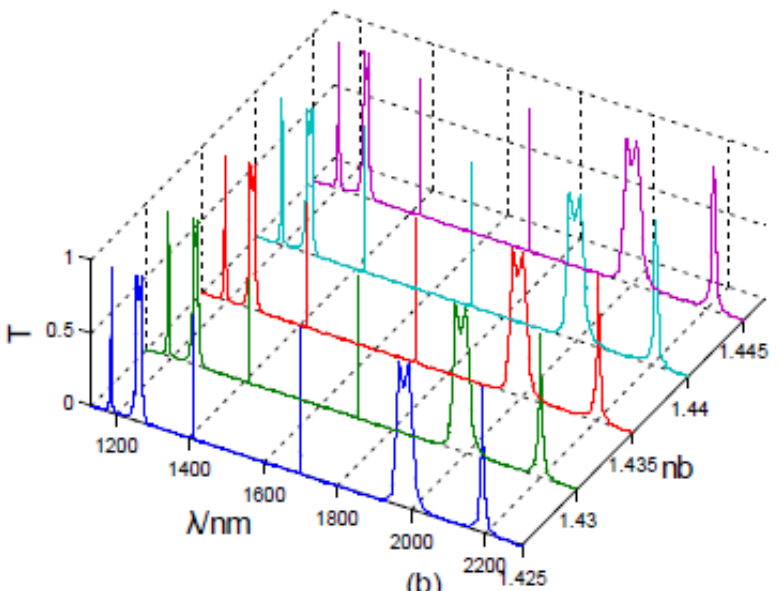

(b)

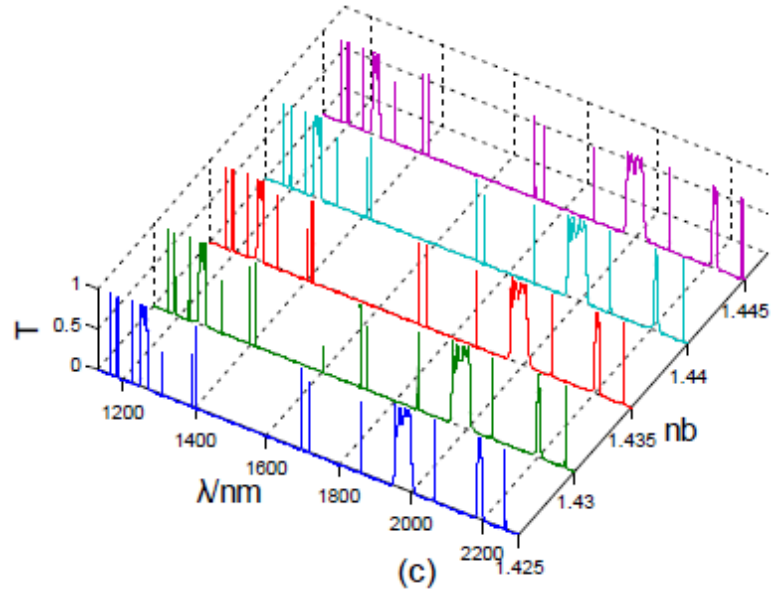

Figure1. The third, fourth and fifth generation transmission spectrum of One-dimensional Cantor quasicrystal structure containing nanoparticle magnetic fluid

The quality factor of localized modes is defined as $Q=\lambda / \Delta \lambda$, where $\lambda$ is the center wavelength of localized modes, $\Delta \lambda$ is the bandwidth of the half peak. The quality factor of localized modes $\lambda_{1}$ and $\lambda_{2}$ (the localized modes in the middle)of fourth and fifth generation are calculated by the table 1 and table 2 
which show that the higher generation of One-dimensional Cantor quasicrystal structure containing nanoparticle magnetic fluid, the higher of the quality factor of the split localized modes; the quality factor of localized modes decreases with the refractive index of magnetic fluid increasing at the same generation of One-dimensional Cantor quasicrystal structure containing nanoparticle magnetic fluid.

Table 1 The quality factor of fourth generation localized modes

\begin{tabular}{ccccccc}
\hline $\mathrm{n}_{\mathrm{b}}$ & $\lambda_{1} / \mathrm{nm}$ & $\Delta \lambda \mathrm{nm}$ & $\mathrm{Q}$ & $\lambda_{2} / \mathrm{nm}$ & $\Delta \lambda \mathrm{nm}$ & $\mathrm{Q}$ \\
\hline 1.425 & 1407.27 & 0.36 & 3909.08 & 1699.20 & 0.51 & 3331.76 \\
1.430 & 1411.82 & 0.37 & 3815.73 & 1704.36 & 0.53 & 3215.77 \\
1.435 & 1416.37 & 0.38 & 3727.29 & 1709.50 & 0.55 & 3108.18 \\
1.440 & 1420.91 & 0.39 & 3643.36 & 1714.63 & 0.57 & 3008.12 \\
1.445 & 1425.46 & 0.40 & 3563.65 & 1719.76 & 0.60 & 2866.27 \\
\hline
\end{tabular}

Table 2 The quality factor of fifth generation localized modes

\begin{tabular}{ccccccc}
\hline $\mathrm{n}_{\mathrm{b}}$ & $\lambda_{1} / \mathrm{nm}$ & $\Delta \lambda / \mathrm{nm}$ & $\mathrm{Q}$ & $\lambda_{2} / \mathrm{nm}$ & $\Delta \lambda \mathrm{nm}$ & $\mathrm{Q}$ \\
\hline 1.425 & 1392.42 & 0.007 & 198917.14 & 1719.50 & 0.012 & 143291.67 \\
1.430 & 1397.15 & 0.008 & 174643.75 & 1725.21 & 0.013 & 132708.46 \\
1.435 & 1401.88 & 0.009 & 155764.44 & 1730.93 & 0.014 & 123637.86 \\
1.440 & 1406.60 & 0.010 & 140660.00 & 1736.64 & 0.015 & 115776.00 \\
1.445 & 1411.32 & 0.011 & 128301.82 & 1742.35 & 0.016 & 108896.88 \\
\hline
\end{tabular}

When the refractive index $n_{b}$ of magnetic liquid increases from 1.425 to 1.445 and the One-dimensional Cantor quasicrystal structure containing nanoparticle magnetic fluid within the band gap(from $1130 \mathrm{~nm}$ to $2400 \mathrm{~nm}$ ) ,the wavelength of the third generation localized modes drift from $1260 \mathrm{~nm}$ and $1986 \mathrm{~nm}$ to $1274 \mathrm{~nm}$ and 2003nm respectively, the drift variation of localized modes are $14 \mathrm{~nm}$ and $17 \mathrm{~nm}$; the wavelength of the fourth generation localized modes drift from 1186nm, 1407nm,1699nm and 2194nm to 1201nm, 1425nm, $1720 \mathrm{~nm}$ and $2219 \mathrm{~nm}$ respectively, the drift variation of localized modes are $15 \mathrm{~nm}, 18 \mathrm{~nm}, 21 \mathrm{~nm}$ and $25 \mathrm{~nm}$; the wavelength of the fifth generation localized modes drift from $1166 \mathrm{~nm}, 1227 \mathrm{~nm}, 1313 \mathrm{~nm}, 1392 \mathrm{~nm}$, $1719 \mathrm{~nm}, 1858 \mathrm{~nm}, 2066 \mathrm{~nm}$ and $2265 \mathrm{~nm}$ to $1181 \mathrm{~nm}, 1243 \mathrm{~nm}, 1331 \mathrm{~nm}, 1411 \mathrm{~nm}, 1742 \mathrm{~nm}, 1882 \mathrm{~nm}$, $2092 \mathrm{~nm}$ and $2294 \mathrm{~nm}$ respectively, the drift variation of localized modes are $15 \mathrm{~nm}, 16 \mathrm{~nm}, 18 \mathrm{~nm}, 19 \mathrm{~nm}$, 23nm, 24nm, 26nm and $29 \mathrm{~nm}$. Accordingly, the wavelength of localized modes shift to the long-wave direction with the refractive index of magnetic fluid increasing; the wavelength drift variation decreases with the increasing of the localized modes level at the same generation (the level of $\lambda_{1}>$ the level of $\lambda_{2}$ ).

\section{Conclusion and Discussion}

The transmission spectrum of One-dimensional Cantor quasicrystal structure containing nanoparticle magnetic fluid is calculated by transfer matrix method. The results show that the number of localized modes increases with the generation of One-dimensional Cantor quasicrystal structure containing nanoparticle magnetic fluid increasing; From the transmission spectrum diagram of the third and fourth generation can be found that one localized mode is split out from both sides of each localized modes of the third generation respectively, and the localized mode also can be split out from the fourth generation to the fifth generation; however, the localized modes of the third generation always can appear on the transmission spectrum of the fourth and the fifth generation, the new split localized modes is always based on the localized modes of previous generation, because all of them include the fixed structure of ABABBB; the quality factor of split localized modes gets higher when the generation of One-dimensional Cantor quasicrystal structure containing nanoparticle magnetic fluid increased, we can get that the next generation structure include the 
double structure of the previous generation from the recursive rule: $S_{n}=S_{n-1} B_{n} S_{n-1}, B_{n}=3^{n-1} B_{1}\left(n \geq 1, B_{1}=B\right)$, that makes the photon can have more time to accumulate and interaction in photonic crystals, so the quality factor gets higher; but the quality factor of localized modes decreases with the refractive index of magnetic fluid increasing at the same generation, because the refractive index of magnetic fluid is close to the refractive index of A dielectric layer with the refractive index of magnetic fluid increasing , the difference of refractive index between the two dielectric layers is smaller, so the quality factor gets lower; it has certain guiding significance for the photonic devices such as optical switch, filter in theory. The wavelength of the localized modes shift to the long-wave direction with the refractive index of magnetic fluid increasing, because the light can transmit stably in the crystal of quasiperiodic structure that must abide the standing-wave conditions $n d=k \lambda, n$ is the refraction index of dielectric layer and $d$ is the thickness, so the wavelength $\lambda$ of localized modes increases with the refractive index $n$ of defect layer increasing for the same level of localized modes ( $k$ is certain) when the thickness $d$ is certain. The number of localized modes in fifth generation are more and the distribution are dense especially the localized modes on both sides of the band gap, the quality factor is high,that has certain guiding significance for making multi-channel filter in theory.

\section{Acknowledgement}

This research was supported by the Innovation Fund Designated for Graduate Students of Jiangxi Province under Grant No.YC2014-S414.

\section{References}

[1] D. Shechtman, I. Blech, D. Gratias, et al: 'Metallic phase with long-range orientational order and no translational symmetry’, Phys. Rev. Lett., 1984, 53(20), 1951-1954.

[2] E. L. Albuquerque and M. G. Cottam: 'Theory of elementary excitations in quasiperiodic structures', Physics Reports, 2003, 376(4), 225-337.

[3] C. Fan, G. Wang, J. Huang: 'Magnetocontrollable photonic crystals based on colloidal ferrofluids', Journal of Applied Physics, 2008, 103(9), 094107-1-094107-3.

[4] G. Yu, S. Pu, X. Wang, H. Ji: 'Tunable one-dimensional photonic crystals based on magnetic fluids', Optik, 2012, 124(17), 2713-2715.

[5] P. Zu, C. Chan, T. Gong, et al: 'Magneto-optical fiber sensor based on band gap effect of photonic crystal fiber infiltrated with magnetic fluid’, Appl. Phys. Lett., 2012, 101(24), 241118-1-241118-4.

[6] C. Fan: 'A study of the properties of several kinds of soft matter', $\mathrm{PhD}$ thesis, Fudan University, Shang Hai, China, 2009, 63-67.

[7] Y. Gao, C. Fan, J. Huang: 'The characteristics research of photonics with colloid ferrofluid', Progress in physics, 2010, 30(4), 387-421.

[8] Y. Miao, B. Liu, K. Zhang, et al: 'Temperature tunability of photonic crystal fiber filled with $\mathrm{Fe}_{3} \mathrm{O}_{4}$ nanoparticle fluid’, Appl. Phys. Lett., 2011, 98(2), 021103-1-021103-3.

[9] Y. Zhao, R. -Q. Lv, Y. Ying: 'Hollow-core photonic crystal fiber Fabry-Perot sensor for magnetic field measurement based on magnetic fluid’, Optics \& Laser Technology, 2012, 44(4), 899-902.

[10] Y. Zhao, Y. Zhang, Q. Wang, et al: 'Photonic crystal fibers Bragg grating filled magnetic fluid for magnetic fields sensing’, Sensor Letters, 2012, 10(1/2), 465-47.

[11] Y. Zhang and D. Li: 'Analysis of Birefringent characteristics of photonic crystal fibers filled magnetic fluid', Lecture Notes in Computer Science and Advances in Brain Inspired Cognitive Systems, 2012, 7366, 250-258. 
[12] T. King and C. Wu: 'Design of multichannel filters based on the use of periodic Cantor dielectric One-dimensionals', Applied optics, 2014, 53(29), 6749-6755.

[13] D. Lenz, C. Seifert, P. Stollmann: 'Zero measure Cantor spectra for continuum one-dimensional quasicrystal', Journal of Differential Equations, 2014, 256(6), 1905-1926.

[14] Y. Xie: 'A study on the Interrelated Questions for the physical Mechanism of Lasing in Random Media', $\mathrm{PhD}$ thesis, Nanchang University, Nan Chang, China, 2007. 